\title{
Is Oxidative Stress a Consequence of Hyperglycemia? Or is Hyperglycemia the Consequence of Oxidative Stress? Or are Both Caused by Insulin Resistance?
}

\author{
Ihsan Boyacı $^{1^{*}}\left(\mathbb{D}\right.$, Türkan Yiğitbaşı ${ }^{2}$ and Handan Ankaralı ${ }^{3}$ \\ ${ }^{1}$ Department of Internal Medicine, İstanbul Medipol University, Turkey \\ ${ }^{2}$ Department of Medical Biochemistry, İstanbul Medipol University, Turkey \\ ${ }^{3}$ Department of Biostatistics and Medical Informatics, Istanbul Medeniyet University, Turkey
}

*Corresponding author: Ihsan Boyacl, Department of Internal Medicine, Istanbul Medipol University, Fatih, Istanbul 34093, Turkey, Tel: 90-532-583-33-39, Fax: 90-212-521-27-83

\begin{abstract}
Objective: The oxidative status may not only be a cause of diabetes, but also a consequence of diabetes. We aimed to evaluate the relationship between hyperglycemia levels and oxidative status in patients with type 2 diabetes using Total Antioxidant Capacity (TAC), Total Oxidative State (TOS) and Oxidative Stress İndex (OSI).

Method: In this study, 145 patients diagnosed with prediabetes $(n=28)$ and type 2 diabetes $(n=117)$ according to $\mathrm{HbA} 1 \mathrm{c}$ and oral glucose tolerance test (OGTT) levels were divided into five groups. The control group $(n=24)$ was composed of healthy individuals. HbA1c was determined by boronate affinity technology according to the NGSP standard. Serum TAC and TOS levels were measured by the colorimetric method.

Results: The TAC was significantly higher in the control group than the other groups $(p=0.001)$, the TOS was significantly higher in $\mathrm{Gr} \mathrm{B}, \mathrm{Gr} \mathrm{C}$ and $\mathrm{Gr} \mathrm{D}$ compared to the other groups $(p=0.004)$, and the OSI was significantly lower in the control group compared to the other groups $(p$ $=0.001)$. A significant positive correlation was indicated between TOS and insulin resistance $(p=0.001)$. There was found a significant positive correlation between TOS and HbA1c $(p=0.005)$.

Conclusions: Increased glycaemia increases TOS in diabetic patients. The main reason for the increase in TOS may be insulin resistance, although, whether it is the cause or the consequence, is ambiguous. It can be claimed that acute glucose fluctuations are more effective in oxidative stress than chronic hyperglycemia.
\end{abstract}

\section{Keywords}

Type 2 diabetes mellitus, Hyperglycemia, Insulin resistance, Total antioxidant capacity, Total oxidative state, Oxidative stress index

\begin{abstract}
Abbreviations
TAC: Total Antioxidant Capacity; TOS: Total Oxidative State; OSI: Oxidative Stress Index; OGTT: Oral Glucose Tolerance Test; T2D: Type 2 Diabetes Mellitus; IR: Insulin Resistance; OS: Oxidative Stress; ROS: Reactive Oxygen Species; HbA1c: Haemoglobin A1c; PD: Prediabetes; HC: Healthy Control; IFG: Impaired Fasting Glucose; FBG: Fasting Blood Glucose; IGT: İmpaired Glucose Tolerance; Gr A: Group A; Gr B: Group B; Gr C: Group C; Gr D: Group D; BMI: Body Mass İndex; WHR: Waist-Hip Ratio; PPG: Postprandial Glucose; NGSP: National Glycohemoglobin Standardization Program; HOMA-IR: Homeostatic Model OfassessmentInsulin Resistance; TG: Triglyceride; Tcol: Total Cholesterol; HDL-c: High Density Lipoprotein cholesterol; LDL-c: LowDensity Lipoprotein Cholesterol; CRP: c-Reactive Protein; mean \pm SD: Mean and Standard Deviation; MS: Metabolic Syndrome; CVD: Cardiovascular Diseases
\end{abstract}

\section{Introduction}

Type 2 diabetes mellitus (T2D) is a disease characterized by insulin deficiency or İnsulin Resistance (IR). In the development of glucose intolerance, IRand $\beta$ cell failure in the muscle/liver represent the main defects. In addition to these, IR and neurotransmitter dysregulation in the brain, accelerated lipolysis,

Citation: Boyacı I, Yiğitbaşı T, Ankaralı H (2021) Is Oxidative Stress a Consequence of Hyperglycemia? Or is Hyperglycemia the Consequence of Oxidative Stress? Or are Both Caused by Insulin Resistance?. Int Arch Endocrinol Clin Res 7:023. doi.org/10.23937/2572-407X.1510023

Accepted: July 17, 2021: Published: July 19, 2021

Copyright: (C) 2021 Boyacı I, et al. This is an open-access article distributed under the terms of the Creative Commons Attribution License, which permits unrestricted use, distribution, and reproduction in any medium, provided the original author and source are credited. 
incretin deficiency/resistance, hyperglucagonemia, increased glucose reabsorption play important roles [1]. Diabetes has the potential to cause a multitude of lifethreatening health complications. Worldwide, diabetes is responsible for $11.3 \%$ of all deaths. Almost half of these deaths are among people under 60 years of age [2]. The seriousness of this picture raises efforts to study the etiopathogenesis and treatment of diabetes in all areas.

In a healthy body, there is a balance between prooxidants and antioxidants. The imbalance in favour of free radicals between free radical formation and antioxidant defence system is called Oxidative Stress (OS) [3]. When OS occurs, Reactive Oxygen Species (ROS) react with various biological targets, especially lipid, protein, carbohydrate and nucleic acids [4,5]. Pancreatic $\beta$ cells contain very low levels of antioxidant enzymes and are therefore highly vulnerable to OS [6]. Many studies have also suggested that $\beta$ cell dysfunction is the result of prolonged exposure to high glucose and high free fatty acidlevel [7]. OS is an important factor in the pathogenesis of many chronic diseases, including diabetes. Recently, it has also been recognized as a key factor in the IR mechanism. Chronic exposure to high glucose, which is the main feature of the diabetic environment, increases ROS production and creates OS $[8,9]$.

For these reasons, attempts to prevent and/or reduce the occurrence of the OS have been recommended as part of treatment programs for patients with T2D. In studies of vitamin $E$ and $C$ on diabetic patients, it has been argued that it lowers blood sugar levels, improves oxidative status parameters, and reducedhemoglobin A1c (HbA1c) [10-12]. It has also been suggested that oral administration of $\alpha$-lipoic acid reduces lipid peroxidation and improves glycometabolic control [13].

The relationships between antioxidant status and the prevention, regulation, and development of diabetes are still debated. Do antioxidant treatments bring new treatment strategies to diabetes? It's unknown, but in this study; We aim to investigate whether the relationship between oxidative status and diabetes severity is significant in patients with T2D. It may also be important for the prediction that persistent hyperglycemia secondary to IR may cause OS and contribute to $\beta$ cell destruction in T2D. The focus of this study will be the role of OS in IR and diabetes.

\section{Material and Method}

\section{Study protocol and participants}

The study consisted of 169 people who applied to the internal medicine outpatient clinic of Istanbul Medipol University Medical Faculty, including prediabetes (PD) $(n=28)$, overt T2D $(n=117)$, and healthy control $(H C)$ ( $n$ $=24$ ). Participants ( 81 females, 88 males) aged from 40 to 70 years were enrolled based on $\mathrm{HbA} 1 \mathrm{c}$ and $75 \mathrm{~g}$ Oral Glucose Tolerance Test (OGTT).

\section{Inclusion criteria}

Healthy volunteers were recruited from those with HbA1c levels lower than $5.7 \%$ and normal OGTT. The people, whose $\mathrm{HbA} 1 \mathrm{c}$ values were $5.7 \% \leq \mathrm{HbA} 1 \mathrm{c} \leq$ $6.4 \%$, and/or who had Impaired Fasting Glucose (IFG) [Fasting Blood Glucose (FBG) levels $100 \mathrm{mg} / \mathrm{dl}$ to 125 $\mathrm{mg} / \mathrm{dl}]$, and/or had İmpaired Glucose Tolerance (IGT) [2-h values in the OGTT of $140 \mathrm{mg} / \mathrm{dl}$ to $199 \mathrm{mg} / \mathrm{dl}$ ], were enrolled to PD group. Diabetic participants were classified according to their HbA1c levels; group A (Gr A) $(\mathrm{HbA} 1 \mathrm{c} \leq 6.4 \%)$, group $\mathrm{B}(\mathrm{Gr} B)(6.5 \% \leq \mathrm{HbA} 1 \mathrm{c} \leq 7.9 \%)$, group $\mathrm{C}(\mathrm{Gr} \mathrm{C})(8.0 \% \leq \mathrm{HbA} 1 \mathrm{c} \leq 9.9 \%)$ and group $\mathrm{D}(\mathrm{Gr}$ D) $(10.0 \% \leq \mathrm{HbA} 1 \mathrm{c})$. The $\mathrm{Gr}$ A patients were individuals who were under control with diabetic agents and had $\mathrm{HbA} 1 \mathrm{c} \leq 6.4 \%$. The diagnosis of T2D was made based on the criteria of the American Diabetes Association (ADA) [14].

\section{Exclusion criteria}

Patients with a cancer diagnosis or cancer treatment, those with immunosuppressive therapy, malnutrition, cirrhosis, anemia, haemolysis patients and pregnant women were not included in the study. The treatments of those taking antidiabetic agents were not intervened. Those taking antioxidants were not included in the study. The study was approved by the Ethics Committee and each participant gave informed consent (No. 10840098604.01.01.E.9284).

\section{Screening}

Participants' age, gender and diabetes duration, medical history and treatment were recorded. Physical examinations were performed and anthropometric measurements were taken. Individuals were classified according to their degree of obesity measured by Body Mass Index (BMI). The standard Waist-to-Hip Ratio (WHR) was accepted as $<1 \mathrm{~cm}$ for men and $<0.8 \mathrm{~cm}$ for women.

\section{Blood analysis}

Blood and spot urine samples of all the study subjects were collected after at least 10 hours of fasting overnight. Blood specimens were allocated in $8.5 \mathrm{ml}$ vacutainers (Becton Dickinson) tubes for biochemical parameters. $\mathrm{HbA} 1 \mathrm{c}$ samples were divided into $2 \mathrm{ml}$ tubes, including Ethylene Diamine Tetra-Acetic Acid (EDTA). Two hours after breakfast, blood samples were taken again for Postprandial Glucose (PPG) levels. The samples were kept at $-80{ }^{\circ} \mathrm{C}$ until analysis. $\mathrm{HbA} 1 \mathrm{c}$ concentration was determined by boronate affinity technology (Quo-Lab ${ }^{\circ}$, EKF Diagnostics PLC, Cardiff, UK) and expressed as a percentage according to the National Glycohemoglobin Standardization Program (NGSP). Plasma glucose was measured by the glucose oxidase method for diagnostic 
criteria. IFG and/or IGT were determined with standard 75 -g glucose OGTT. The insulin, fasting level was measured by Cobas e411 and C-peptide fasting was analyzed by ADVIA Centaur XP (Siemens). To detect the haHomeostatic Model of Assessment-Insulin Resistance (HOMA-IR) the following formula was used fasting insulinlevel $(\mu \mathrm{U} / \mathrm{ml}) \times$ fasting glucose level $(\mathrm{mg} / \mathrm{dl}) / 405$. FBG, PPG, triglyceride (TG), total cholesterol (Tcol), high-density lipoprotein cholesterol (HDL-C), LowDensity Lipoprotein cholesterol (LDL-C), creatinine and uric acid levels were measured by VITROS 350 Chemical Systems (Ortho Clinical Diagnostics). C-Reactive Protein (CRP) and microalbumin, that in spot urine, were tested with the use of an i-chroma reader device (Boditech).

\section{Total oxidant assay}

The TAC value was measured in serum using the colorimetric method [15]. ABTS [2,2'-azino-bis(3ethyl- benzothiazoline-6-sulfonic acid)] (SigmaAldrich, Taufkirchen, Germany) reagent is radicalized by hydrogen peroxide (Sigma-Aldrich, Taufkirchen, Germany). When serum is added, antioxidants in the serum neutralize existing ABTS radicals. The absorbance is measured at $658 \mathrm{~nm}$.

\section{Total antioxidant assay}

TOS value was measured in serum using the colorimetric method [16]. Fe2SO4 dissolves in water, releasing $\mathrm{Fe}^{2+}$. Oxidants found in serum enable $\mathrm{Fe}^{2+}$ to $\mathrm{Fe}^{3+}$ oxidation. The X-orange (Sigma-Aldrich, Taufkirchen, Germany) reagent used gives a colored complex with $\mathrm{Fe}^{3+}$. The absorbance was measured at $658 \mathrm{~nm}$. OSI is calculated by the formula (TOS/TAC) $\times 100$.

\section{Statistical analysis}

The primary aim of the study was to classify five patient groups and one $\mathrm{HC}$ group, according to $\mathrm{HbA1c}$ and OGTT; to determine whether they differ in terms of TAC, TOS and OSI. Appropriateness of numerical variables in normal distribution that was observed during the study was evaluated by One-sample Kolmogorov Smirnov test and the identifying values were calculated as the mean and standard deviation (mean \pm SD). Taking account of the distribution of the values, the one-way ANOVA model and Kruskal Wallis test were used in a comparison of the groups, and then different groups were detected via post-hoc Scheffe test. The relevance between the values was observed by Pearson's correlation coefficient. The statistical significance level was considered as $p<0.05$ and SPSS V22.0 statistical software (SPSS, Inc, USA) was used.

\section{Results}

The HC group consists of 24 (14.2\%) individuals, PD and diabetes patients 145 (85.8\%), in total 169 participants. According to all groups, a significant difference was found between the ages of individuals,
BMI (Body Mass Index) and WHR (Waist-Hip Ratio). In all groups; There was also a significant difference in terms of HbA1c, FBG, PPG, fasting insulin, IR, CRP, C-peptide, uric acid, TAC, TOS and OSI. There was no difference in terms of IFG and T col. The details of all participants are shown in Table 1.

The TAC level was significantly higher in the healthy group than the other groups $(p=0.001)$, the TOS level was significantly higher in $\mathrm{Gr} \mathrm{B}, \mathrm{Gr}$ C and Gr D compared to the other groups $(p=0.004)$. OSI was significantly lower in the HC group compared to the other groups ( $p$ $=0.001$ ) (Table 2).

Once all individuals were considered, no significant association was found between $\mathrm{HbA} 1 \mathrm{c}$ and TAC. There was a significant positive correlation between $\mathrm{HbA1c}$ and TOS and between HbA1c and OSI. When the groups were examined separately, there was no significant relationship between $\mathrm{HbA} 1 \mathrm{c}$ level and TOS and OSI. FBG and PPG, it was seen that both parameters showed a significant positive correlation with TOS and OSI levels. No significant correlation was found between the TAC level and both parameters. There was a significant positive correlation between PPG and TOS in the PD group (Table 3).

In our study, it was revealed that there is a significant negative relationship between TAC and C-peptide level. In contrast, a significant positive correlation was found between TOS and C-peptide as well as a positive correlation between OSI and C-peptide level. On the other hand, although there was no significant correlation between IR and TAC, a significant positive correlation was found between IR and TOS. In addition, there was a significant positive correlation between IR and OSI. In patients with $\mathrm{Gr} D$, there was a significant negative correlation between TAC and IR, as well as a significant positive correlation between OSI and IR (Table 3).

CRP is an indicator of subclinical inflammation. Although there was a significant positive correlation between CRP and HbA1c, contrary to expectations no significant correlation was found between CRP level and TAC, TOS and OSI. Additionally, a significant negative correlation was revealed between uric acid level and $\mathrm{HbA1c}$. A significant negative correlation was detected between microalbuminuria level and TAC and a positive correlation with OSI. Moreover, there was a significant positive correlation between TAC and HDL-c level. There was a significant positive correlation between OSI and Tcol, as well as a significant positive correlation between TG (Table 4).

In the anthropoemetric examinations of individuals, a significant relationship was found between BMI with TAC, TOS and OSI levels. On the contrary, there was no significant relationship between the oxidation state parameters and WHR (Table 5). No significant 


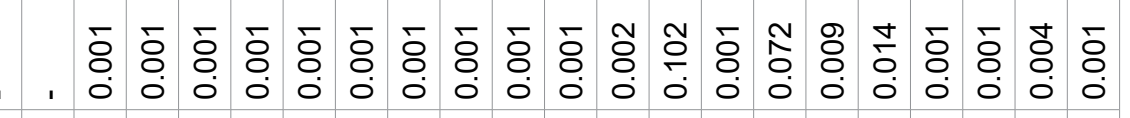

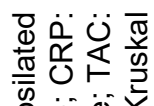

ฉ.

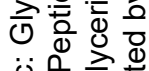

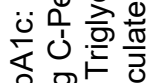

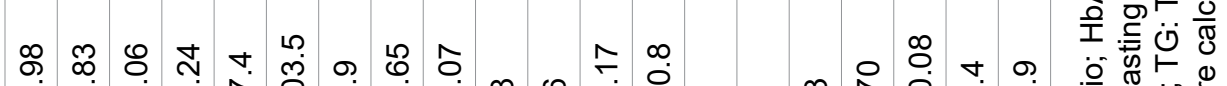

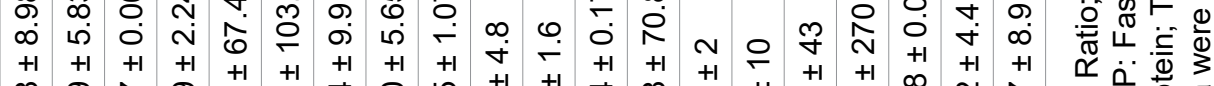

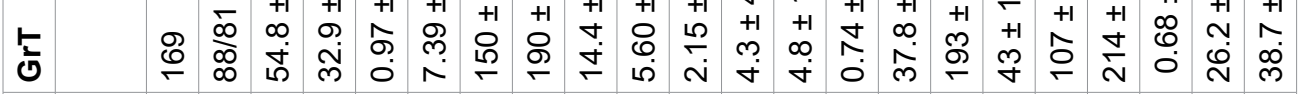

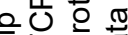

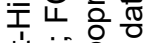

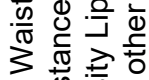

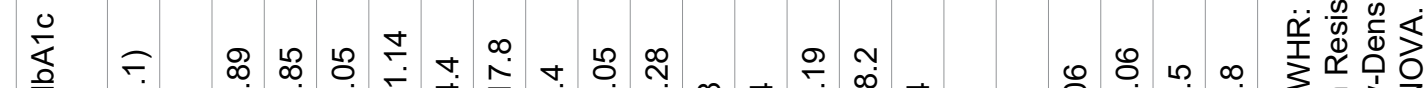

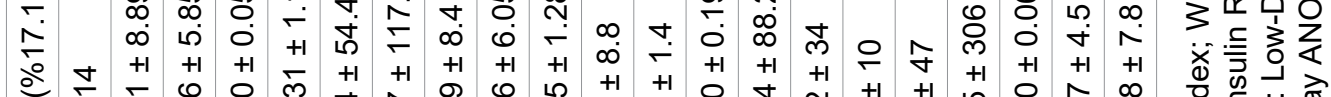

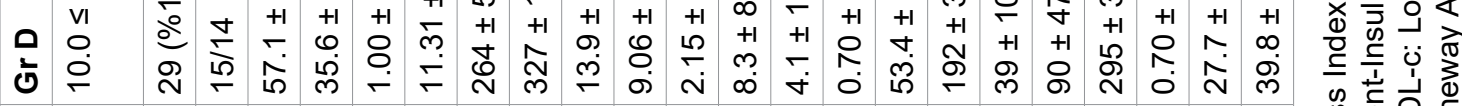

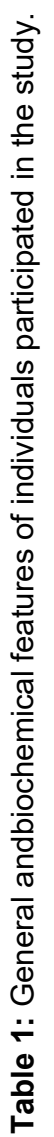

$a$

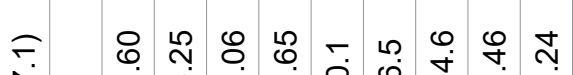

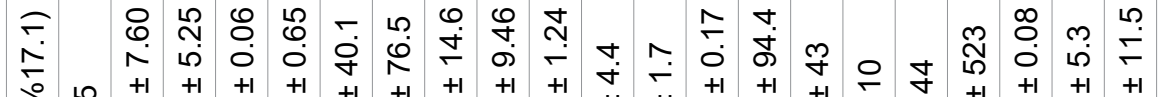

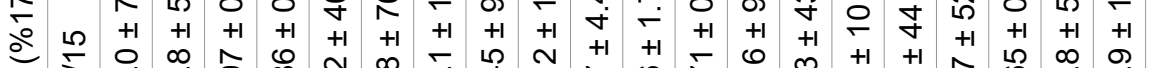

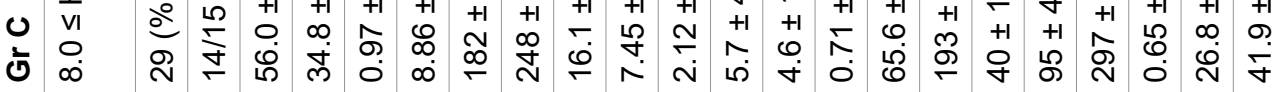

ू.

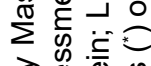

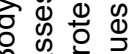

这茯

$\sum \frac{0}{1}$

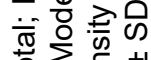

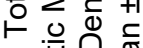

$\stackrel{\substack{1 \\ 0}}{2}$

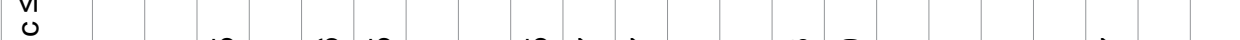

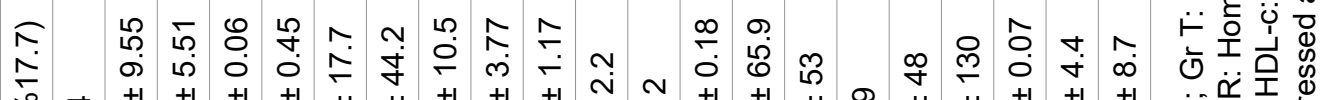

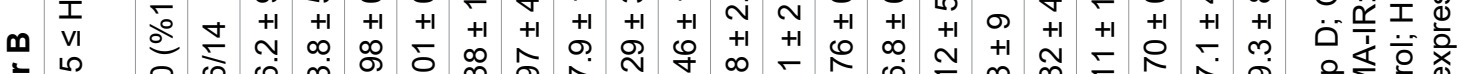

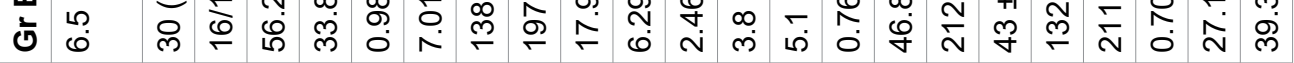

$+\frac{0}{2}$

O

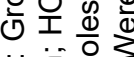

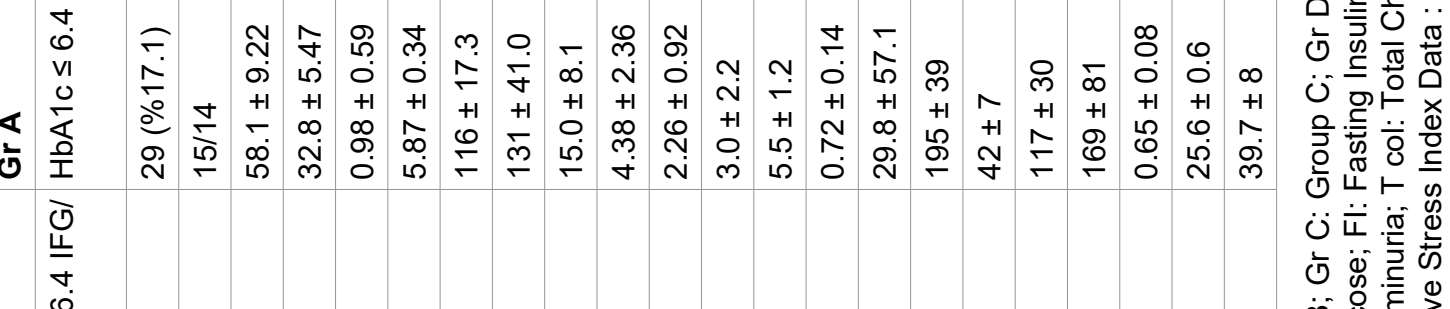

vit

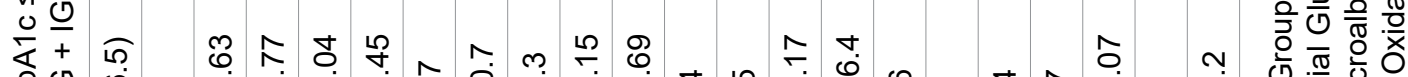

全岱

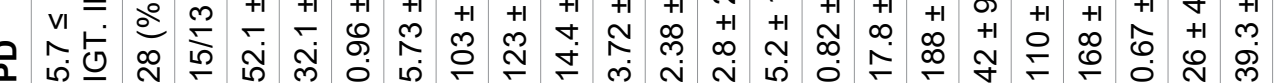

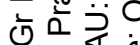

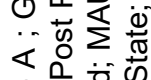

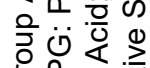

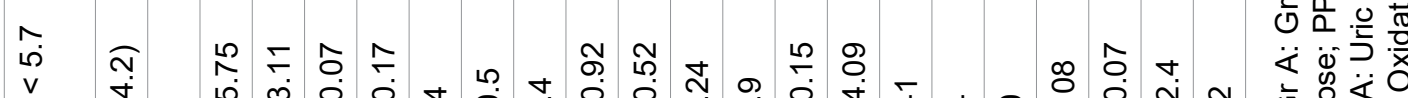

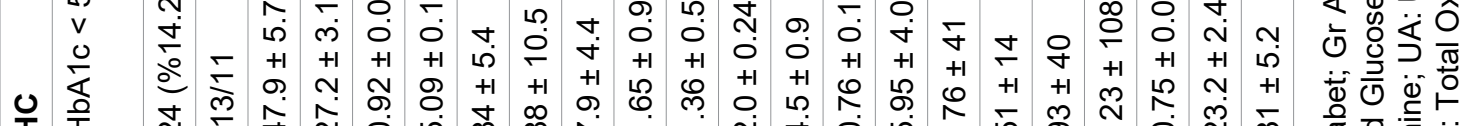

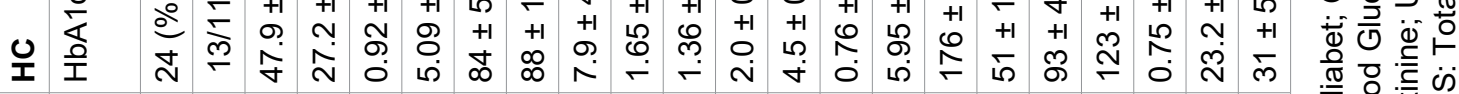
중 을

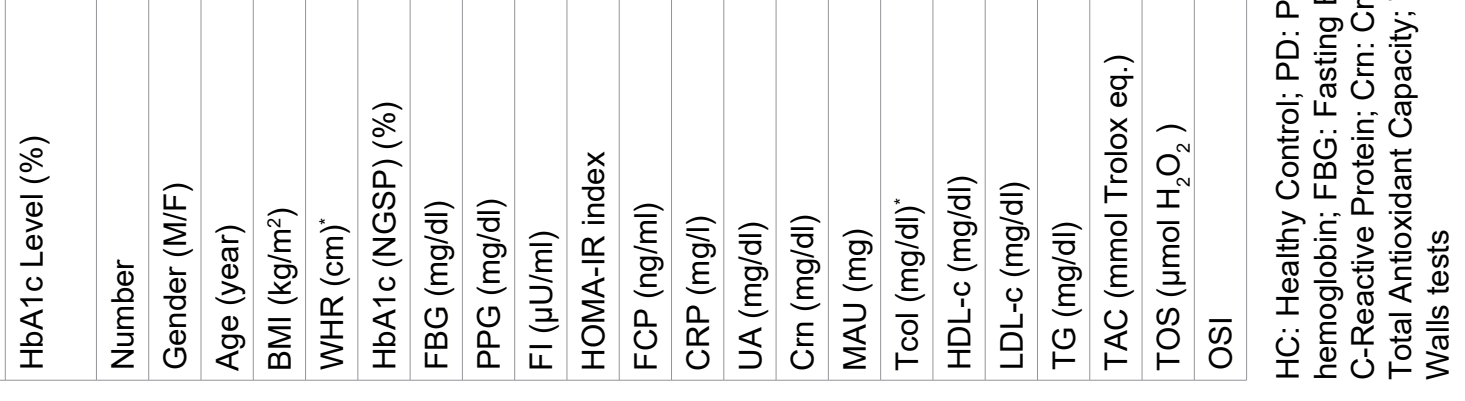


Table 2: Distribution of TAC. TOS and OSI by groups.

\begin{tabular}{|c|c|c|c|c|c|c|c|c|c|c|c|c|}
\hline & \multicolumn{3}{|c|}{ TAC (mmol Trolox eq.) } & \multirow[t]{2}{*}{ p } & \multicolumn{3}{|c|}{$\operatorname{TOS}\left(\mu \mathrm{mol} \mathrm{H}_{2} \mathrm{O}_{2}\right)$} & \multirow[t]{2}{*}{ p } & \multicolumn{3}{|c|}{ OSI } & \multirow[t]{2}{*}{$p$} \\
\hline & $N$ & Mean & SD & & $\mathrm{N}$ & Mean & SD & & $\mathrm{N}$ & Mean & SD & \\
\hline $\mathrm{HC}$ & 24 & $0.75 a$ & 0.07 & \multirow[t]{6}{*}{0.001} & 24 & $23.2 a$ & 2.4 & \multirow[t]{6}{*}{0.004} & 24 & 31.0a & 5.2 & \multirow[t]{6}{*}{0.001} \\
\hline PD & 28 & $0.67 b$ & 0.07 & & 28 & 26.0ab & 4.3 & & 28 & $39.3 b$ & 7.2 & \\
\hline Gr A & 29 & $0.65 b$ & 0.08 & & 29 & 25.6ab & 3.6 & & 29 & $39.7 b$ & 8 & \\
\hline Gr B & 30 & $0.70 \mathrm{~b}$ & 0.07 & & 30 & $27.1 b$ & 4.4 & & 30 & $39.3 b$ & 8.7 & \\
\hline Gr C & 29 & $0.65 b$ & 0.08 & & 29 & $26.8 b$ & 5.3 & & 29 & $41.9 b$ & 11.5 & \\
\hline Gr D & 29 & $0.70 \mathrm{~b}$ & 0.06 & & 29 & $27.7 \mathrm{~b}$ & 4.5 & & 29 & $39.8 \mathrm{~b}$ & 7.8 & \\
\hline
\end{tabular}

If the averages contain completely different letters. they are significantly different from each other. The differences are not significant if there are same or common letter (evaluated by One-Way ANOVA and post hoc Scheffe test.) Data: mean \pm SD; TAC: Total Antioxidant Capacity; TOS: Total Oxidative state; OSI: Oxidative Stress Index; HC: Healthy Control; PD: Prediabet; Gr A: Group A ; Gr B: Group B; Gr C: Group C; Gr D: Group D; Gr T: Group Total

Table 3: Relationship between hyperglycaemia components and oxidative stress parameters*.

\begin{tabular}{|c|c|c|c|c|c|c|}
\hline & \multicolumn{2}{|c|}{ TAC } & \multicolumn{2}{|c|}{ TOS } & \multicolumn{2}{|c|}{ OSI } \\
\hline & $\mathbf{r}$ & p & $\mathbf{r}$ & $\mathbf{p}$ & $\mathbf{r}$ & p \\
\hline HbA1c (NGSP) (\%) & -0.052 & 0.503 & $0.217^{\star}$ & 0.005 & $0.174^{*}$ & 0.024 \\
\hline FBG (mg/dl) & -0.023 & 0.768 & $0.249^{*}$ & 0.001 & $0.179^{*}$ & 0.020 \\
\hline PPG (mg/dl) & -0.079 & 0.316 & $0.230^{*}$ & 0.003 & $0.198^{*}$ & 0.011 \\
\hline $\mathrm{FCP}(\mathrm{ng} / \mathrm{ml})$ & -0.189 & 0.014 & 0.274 & 0.001 & 0.279 & 0.001 \\
\hline HOMA-IR index & -0.142 & 0.067 & $0.319^{*}$ & 0.001 & 0.297 & 0.001 \\
\hline $\mathrm{FI}(\mu \mathrm{U} / \mathrm{ml})$ & -0.220 & 0.004 & $0.288^{*}$ & 0.001 & $0.316^{*}$ & 0.001 \\
\hline
\end{tabular}

HbA1c: Glycosilated Hemoglobin; FBG: Fasting Blood Glucose; PPG: Post Prandial Glucose; FCP: Fasting C-Peptide; HOMA-IR: Homeostatic Model of Assessment-Insulin Resistance; FI: Fasting Insulin; TAC: Total Antioxidant Capacity; TOS: Total Oxidative State; OSI: Oxidative Stress Index *pearson correlation analysis

Table 4: Relation of biochemical parameters to oxidative stress parameters*.

\begin{tabular}{|c|c|c|c|c|c|c|c|c|}
\hline & \multicolumn{2}{|c|}{ HbA1c } & \multicolumn{2}{|c|}{ TAC } & \multicolumn{2}{|c|}{ TOS } & \multicolumn{2}{|c|}{ OSI } \\
\hline & $\mathbf{r}$ & $\mathbf{p}$ & $\mathbf{r}$ & $\mathbf{p}$ & $r$ & $\mathbf{p}$ & $\mathbf{r}$ & $\mathbf{p}$ \\
\hline CRP (mg/l) & $0.409^{*}$ & 0.001 & 0.024 & 0.761 & 0.020 & 0.803 & -0.008 & 0.918 \\
\hline $\mathrm{UA}(\mathrm{mg} / \mathrm{dl})$ & $-0.199^{*}$ & 0.010 & $-0.358^{*}$ & 0.001 & $0.231^{\star}$ & 0.003 & $0.370^{*}$ & 0.001 \\
\hline MAU (mg) & $0.187^{*}$ & 0.016 & $0.176^{\star}$ & 0.024 & $0.172^{*}$ & 0.027 & $0.226^{*}$ & 0.003 \\
\hline Tcol $(\mathrm{mg} / \mathrm{dl})^{*}$ & 0.026 & 0.734 & -0.091 & 0.239 & $0.294^{*}$ & 0.001 & $0.242^{*}$ & 0.002 \\
\hline HDL-c (mg/dl) & $-0.243^{*}$ & 0.002 & $-0.245^{*}$ & 0.001 & $-0.524^{*}$ & 0.001 & $-0.505^{*}$ & 0.001 \\
\hline LDL-c (mg/dl) & -0.151 & 0.051 & -0.098 & 0.208 & -0.151 & 0.051 & -0.059 & 0.446 \\
\hline TG (mg/dl) & $0.223^{*}$ & 0.004 & -0.008 & 0.917 & $0.543^{*}$ & 0.001 & $0.378^{*}$ & 0.001 \\
\hline
\end{tabular}

HbA1c: Glycosilated Hemoglobin; CRP: c-Reactive Protein; UA: Uric Acid; MAU: Microalbuminuria; T col: Total Cholesterol; HDL-c: High-Density Lipoprotein; LDL-c: Low-Density Lipoprotein; TG: Triglyceride; *pearson correlation analysis

Table 5: Comparison of oxidative stress parameters and hyperglycaemia components with anthropoemetric measurements*.

\begin{tabular}{|c|c|c|c|c|c|c|c|c|c|c|}
\hline & \multicolumn{10}{|c|}{ HbA1c (\%) TAC (mmol Trolox eq.) TOS $\left(\mu \mathrm{mol} \mathrm{H}_{2} \mathrm{O}_{2}\right)$ OSIHOMA-IR } \\
\hline & $\mathbf{r}$ & $\mathbf{p}$ & $\mathbf{r}$ & $\mathbf{p}$ & $r$ & $\mathbf{p}$ & $\mathbf{r}$ & $\mathbf{p}$ & $\mathbf{r}$ & $\mathbf{p}$ \\
\hline BMI $\left(\mathrm{kg} / \mathrm{m}^{2}\right)$ & $0.325^{\star}$ & 0.001 & $-0.217^{*}$ & 0.005 & $0.213^{*}$ & 0.006 & $0.259^{*}$ & 0.001 & $0.325^{\star}$ & 0.001 \\
\hline WHR (cm) & $0.219^{*}$ & 0.005 & -0.124 & 0.113 & 0.065 & 0.406 & 0.101 & 0.196 & $0.154^{*}$ & 0.050 \\
\hline
\end{tabular}

HbA1c: Glycosilated Hemoglobin; TAC: Total Antioxidant Capacity; TOS: Total Oxidative State; OSI: Oxidative Stress Index; HOMA-IR: Homeostatic Model of Assessment-Insulin Resistance; BMI: Body Mass Index; WHR: Waist-Hip Ratio * pearson correlation analysis

relationship was found between obesity level with TAC ( $p=0.079$ ), but a significant relationship was revealed between TOS and OSI levels [( $p=0.024) ;(p=0.023)]$ (Table 6).

\section{Discussion}

The oxidation of glucose is considered the principal source of free radicals. On the one hand, hyperglycemia causes free radicals, on the other hand, it is claimed 
Table 6: Distribution of hyperglycemia and oxidative stress parameters according to obesity levels.

\begin{tabular}{|c|c|c|c|c|c|c|c|c|c|c|}
\hline Obesity degree & BMI $\left(\mathrm{kg} / \mathrm{m}^{2}\right)$ & Frequency & HbA1c & p & TAC & p & TOS & $\mathbf{p}$ & OSI & p \\
\hline Normal weight & $18.5-24.9$ & $8(\% 4.7)$ & $5.8 \pm 1.4$ & $0.013^{*}$ & $0.70 \pm 0.12$ & 0.079 & $23.2 \pm 2.7$ & 0.024 & $34.8 \pm 12$ & 0.023 \\
\hline Overweight & $25-29.9$ & $44(\% 26)$ & $6.9 \pm 2.4$ & & $0.70 \pm 0.08$ & & $24.8 \pm 3.5$ & & $35.9 \pm 8$ & \\
\hline $1^{\circ}$ obese & $30-34.9$ & $53(\% 31.4)$ & $7.2 \pm 2.0$ & & $0.69 \pm 0.07$ & & $26.3 \pm 4.2$ & & $38.2 \pm 8.2$ & \\
\hline $2^{\circ}$ obese & $35-39.9$ & $39(\% 23.1)$ & $8.0 \pm 2.0$ & & $0.67 \pm 0.07$ & & $27.3 \pm 4.9$ & & $40.8 \pm 8.7$ & \\
\hline Morbid obese & $40 \leq$ & $23(\% 13.6)$ & $8.2 \pm 2.3$ & & $0.68 \pm 0.08$ & & $26.8 \pm 4.5$ & & $41.9 \pm 9.2$ & \\
\hline
\end{tabular}

BMI: Body Mass Index; HbA1c: Glycosilated Hemoglobin; TAC: Total Antioxidant Capacity; TOS: Total Oxidative State; OSI: Oxidative Stress Index. evaluated by One-Way ANOVA and post hoc Scheffe test.) Data: mean \pm SD

that the endogenous antioxidant defense system is disrupted in many ways during diabetes. While some articles stated that TAC decreased in T2D, another reported that they observed an increase $[6,9,17,18]$. In a study of patients with Metabolic Syndrome (MS), they found a significant reduction in TAC in MS compared to normal subjects, but this decrease was not seen among normal and diabetic subjects with MS [19]. However, there have been some arguing that OS contributes to the formation of atherosclerotic plaques and the increased risk of Cardiovascular Disease (CVD) in diabetic patients [20].

In our study, we took the HbA1c and OGTT levels as the hyperglycemia level and divided them into groups based on this. The TAC level was found to be significantly higher in the $\mathrm{HC}$ group compared to the diabetic groups, but no significant difference was found between the diabetes groups. TOS and OSI increased significantly as the severity of diabetes increased, in other words, as the regulation deteriorated. Although we did not find a significant relationship between $\mathrm{HbA} 1 \mathrm{c}$ level and TAC levels, we found a significant positive relationship between $\mathrm{HbA} 1 \mathrm{c}$ level and TOS and OSI.In one study, they processed the thesis that there is an inverse relationship between TAC and FBG and that oxidative damage due to hyperglycemia may have contributed to lower antioxidant levels [18]. In another study, they suggested that PPG and acute glucose fluctuations increase OS more than chronic hyperglycemia [21].

In our data, although FBG and PPG showed a significant positive correlation with TOS and OSI levels, there was no significant relationship between both parameters and TAC levels. In addition, when only the PD group was studied, there was a significant positive relationship between PPG and TOS. Based on these data, TAC is significantly higher in the HC group than in diabetics but does not change with the severity of diabetes. The TAS level increases gradually starting from the prediabetes period with the increase of the glycaemic level, and this causes an increase in OSI. TAC cannot adequately respond to the increase in TAS level. It this also supports the thesis that acute glucose fluctuations are more effective on OS than chronic hyperglycemia.

Oxidative stress appears to be more alarming in metabolic disorders, particularly T2D. It has been discussed in many studies that it contributes to the progression of diabetes by causing impairment of insulin action $[9,22]$. In the early stage, IR is compensated by hyperinsulinemia so that normal glucose tolerance is maintained. Increased IR or decreased insulin secretion or both advances the process towards IGT [23].

C-peptide is produced in Langerhans pancreatic $\beta$ cells and is the best measure of endogenous insulin secretion [24]. In our study, we found a positive significant relationship between fasting insulin, IR, C-peptide and TOS, OSI. A significant negative correlation was shown between the TAC level and C-peptide and insulin fasting. We could not find a correlation between $\mathrm{HbA} 1 \mathrm{c}$ and $\mathrm{C}$-peptide. We revealed a significant positive correlation between C-peptide and FBG as well as between C-peptide and PPG. The reason for this may be $\mathrm{IR}$, although $\beta$ cells produce insulin, here it compensates by $C$-peptide increase and hyperinsulinemia as there is resistance to insulin on the receptor basis. Therefore, hyperinsulinemia causes an increase in OS. This view supports the argument that insulin needs is compensated by the increase in C-peptide at an early stage. Thus, contrary to popular belief, the increase in C-peptide may not always contribute positively to the course of diabetes. Furthermore, it can also be argued that acute glucose fluctuations (FBG and PPG) are more effective on IR and OS than chronic hyperglycemia.

Although uric acid is an important antioxidant in human plasma, it has been suggested as a prooxidant in the development of obesity, hypertension, andCVD, which are conditions associated with OS [25]. This paradox is explained by the thesis that it can function as an antioxidant in plasma and a prooxidant in the cell [26]. In this study, uric acid level showed a significant negative correlation with $\mathrm{HbA1C}, \mathrm{FBG}$ and PPG. It also showed a significant positive correlation with uric acid, TOS, OSI, fasting insulin and C-peptide, while it showed a significant negative correlation with TAC. When we examined our data, the oxidant-antioxidant paradox was seen here as well. Whereas we expected a parallel between all the parameters of hyperglycemia and uricaemia, we saw the opposite.

Obesity has been reported as a strong and independent indicator of systemic OS. Weight gain 
causes an increase in adipose tissue mass and damage to adipocytes, initiation of inflammatory processes and entry of macrophages into adipose tissue [27]. These change may stimulate the entry of proinflammatory cytokines, adipokines, fatty acids into the systemic circulation and triggering OS [20]. It has been shown that $\mathrm{BMI}, \mathrm{WHR}$ is associated with T2D. It has been suggested that central obesity (WHR) is more diabetogenic than general obesity (BMI) [28]. In our data, we found that $\mathrm{BMI}$ and WHR were significantly positively correlated with $\mathrm{HbA1c}, \mathrm{IR}$, FBG and PPG (Table 5). Although the $\mathrm{BMI}$ of the participants was negatively correlated with TAC, positively correlated with TOS and OSI levels, no significant relationship was found with WHR. Contrary to expectations, it can be said that general obesity is more effective than central obesity on antioxidant capacity. In addition, although there was a positive correlation between $\mathrm{BMI}$ and CRP, no correlation was found between WHR and CRP. Hence, he may think that subclinical inflammation is more common in general obesity than central obesity. There was no significant relationship between obesity level and TAC ( $p=0.079)$, but a significant relationship was observed with TOS and OSI ([p = 0.024]; [ $p=0.023]$ ) (Table 6). It was observed that the antioxidant status was significantly improved in morbidly obese patients with T2D who underwent bariatric surgery [29]. As a result, OS mediates the relationship between $\mathrm{BMI}$ and $\mathrm{HbA1c}$. Obesity may be the cause of systemic OS leading to the increased glycation of haemoglobin independent of glucose levels. Hence, the $\mathrm{HbA} 1 \mathrm{c}$ level may rise disproportionately at a given glycaemic level in obese individuals. It has also been suggested that $\mathrm{HbA} 1 \mathrm{c}$ does not always reflect the true glucose concentration in obese individuals [30].

Diabetic nephropathy is a microvascular complication and is the most common cause of end-stage renal disease. The earliest marker of diabetic nephropathy is microalbuminuria. We determined a negative correlation between microalbuminuria level and TAC, a positive relationship between TOS and OSI (Table 6). There was also a positive correlation between microalbuminuria level and HbA1c, FBG, PPG and IR. While microalbuminuria correlated positively with $\mathrm{BMI}$, there was no correlation with WHR. The OS has been shown as the cause of diabetic nephropathy [31,32]. Our data also confirm that increased OS will contribute to the pathogenesis of diabetic nephropathy.

Dyslipidemia in diabetes is associated with hypertriglyceridemia, low HDL-C and high LDL-C. Peroxidation and dysfunction of HDL-c occur due to the OS in T2D patients. As a result, loss of antiatherogenic activity, impairment of antioxidative capacity and endothelial dysfunction occur. Peroxidation of LDL-C 'is inhibited in the presence of HDL-c [20,33,34]. In our study, positiverelationship was found between HDL-c level and TAC, and a significant negative correlation with TOS and OSI. There was a significant correlation between T col, TG with TOS and OSI (Table 4). HDL-C has a great capacity to protect LDL-c from oxidative modification, but its potential therapeutic use to prevent atherosclerosis is not yet complete. Indeed, this supports that uncertainty about what role HDL-c will play in treatment strategies remains.

\section{Conclusion}

In diabetics, we found that, although TAC remained constant, TOS decreased as glycemia increased. We also observed that TAC decreased in diabetics compared to healthy controls. Therefore, we can argue that hyperglycemia is the primary trigger factor. It may also be suggested that acute glucose fluctuations are more effective than chronic hyperglycemia in oxidative stress. The main mechanism in the pathophysiology of T2D is insulin resistance. Insulin resistance brings along hyperinsulinemia. Hyperinsulinemia causes $\beta$ cell loss by creating oxidative stress and thus insulin insufficiency/ deficiency occurs. According to these results, breaking and preventing insulin resistance in T2D should be the priority of our treatment strategies. On the other hand, it is unfortunately difficult to reveal the cause-effect relationship in such cross-sectional studies.

While creating LDL-c lowering therapies, we should develop balancing treatment options for HDL-C and also not ignore hypertriglyceridemia treatment options. Contrary to expectations, it can be said that general obesity is more effective on antioxidant capacity than central obesity.

The uric acid prooxidant-antioxidant paradox is still a closed box that needs to be studied. Finally, recommending intake of supplements to reduce oxidative stress and developing treatments in this regard may both control T2D and prevent its complications.

\section{Funding}

This research did not receive any specific grant from any funding agency in the public, commercial or not-forprofit sector.

\section{Conflict of Interest}

The authors declare that there is no conflict of interest that could be perceived as influencing the objectivity of this review.

\section{Acknowledgements}

The authors thank Dr. Sevinç Beşışık for initial collaboration on the manuscript.

\section{References}

1. DeFronzo RA, Eldor R, Abdul-Ghani M (2013) Pathophysiologic Approach to Therapy in Patients With Newly Diagnosed Type 2 Diabetes. Diabetes Care 36: 127138.

2. International Diabetes Atlas (2019) Brussels: IDF. 
3. Evans JL, Goldfine ID, Maddux BA, Grodsky GM (2002) Oxidative stress and stress-activated signaling pathways: A unifying hypothesis of type 2 diabetes. Endocr Rev 23: 599-622.

4. Maritim AC, Sanders RA, Watkins JB (2003) Diabetes, oxidative stress, and antioxidants: A review. J Biochem Mol Toxicol 17: 24-38.

5. Tiwari BK, Pandey KB, Abidi AB, Rizvi SI (2013) Markers of Oxidative Stress during Diabetes Mellitus. J Biomark 2013: 378790.

6. Savu O, Ionescu-Tirgoviste C, Atanasiu V, Gaman L, Papacocea R, et al. (2012) Increase in total antioxidant capacity of plasma despite high levels of oxidative stress in uncomplicated type 2 diabetes mellitus. J Int Med Res 40: 709-716.

7. Evans JL, Ira D, Goldfine ID, Maddux BA. Grodsky GM (2003) Are Oxidative Stress Activated Signaling Pathways Mediators of Insulin Resistance and $\beta$-Cell Dysfunction? Diabetes 52: 1-8.

8. Hurrle S, Hsu WH (2017) The etiology of oxidative stress in insulin resistance. Biomed J 40: 257-262.

9. Maslov LN, Naryzhnaya NV, Boshchenko AA, Popov SV, Ivanov VV, et al. (2019) Is oxidative stress of adipocytes a cause or a consequence of the metabolic syndrome? J Clin Transl Endocrinol 15: 1-5.

10. Shinde SN, Dhadke VN, Suryakar AN (2011) Evaluation of oxidative stress in type 2 diabetes mellitus and followup along with vitamin e supplementation. Indian $\mathrm{J}$ Clin Biochem 26: 74-77.

11. Jain AB, Jain VA (2012) Vitamin $E$, its beneficial role in diabetes mellitus (DM) and its complications. J Clin Diagn Res 6: 1624-1628.

12. Rafighi Z, Shiva A, Arab S, Yusuf RM (2013) Association of dietary vitamin $C$ and e intake and antioxidant enzymes in type 2 diabetes mellitus patients. Glob $\mathrm{J}$ Health Sci 5: 183-187.

13. Porasuphatana S, Suddee S, Nartnampong A, Konsil J, Harnwong B, et al. (2012) A Glycemic and oxidative status of patients with type 2 diabetes mellitus following oral administration of alphalipoic acid: A randomized doubleblinded placebocontrolled study. Asia Pac J Clin Nutr 21: 12-21.

14. Chamberlain JJ, Rhinehart AS, Shaefer CF, Neuman A (2016) Diagnosis and Management of Diabetes: Synopsis of the 2016 American Diabetes Association Standards of Medical Care in Diabetes. Ann Intern Med 164: 542-552.

15. Erel O (2004) A novel automated method to measure total antioxidant response against potent free radical reactions. Clin Biochem 37: 112-119.

16. Erel O (2005) A new automated colorimetric method for measuring total oxidant status. Clin Biochem 38: 11031111.

17. Komosińska-Vassev K, Olczyk K, Pawel O, WinszSzczotka K (2005) Effects of metabolic control and vascular complications on indices of oxidative stress in type 2 diabetic patients. Diabetes Res Clin Pract 68: 207-216.

18. Odum EP, Ejilemele AA, Wakwe VC (2012) Antioxidant status of type 2 diabetic patients in Port Harcourt, Nigeria. Niger J Clin Pract 15: 55-58.

19. Hopps E, Canino B, Montana M, Presti RL, Averna MR, et al. (2014) Behavior of the total antioxidant status in a group of subjects with metabolic syndrome. Diabetes Metab Syndr 8: 166-169.
20. Ito F, Sono Y, Ito T (2019) Measurement and clinical significance of lipid peroxidation as a biomarker of oxidative stress: Oxidative stress in diabetes, atherosclerosis, and chronic inflammation. Antioxidants 8: 72.

21. Monnier L, Mas E, Ginet C, Michel F, Villon L, et al. (2006) Activation of oxidative stress by acute glucose fluctuations in patients with Type 2 Diabetes. JAMA 295: 1681-1687.

22. Asmat U, Abad K, Ismail K (2016) Diabetes mellitus and oxidative stress-A concise review. Saudi Pharm J 24: 547553.

23. Reaven GM (1988) Banting lecture 1988. Role of insulin resistance in human disease. Diabetes 37: 1595-1607.

24. Jones AG, Hattersley AT (2013) The clinical utility of C-peptide measurement in the care of patients with diabetes. Diabet Med 30: 803-817.

25. Lippi G, Montagnana M, Franchini M, Favaloro EJ, Targher $\mathrm{G}$, et al. (2008) The paradoxical relationship between serum uric acid and cardiovascular disease. Clinica Chimica Acta 392: 1-7.

26. Sautin YY, Johnson RJ (2008) Uric acid: The oxidantantioxidant paradox. Nucleosides, Nucleotides and Nucleic Acids 27: 608-619.

27. Flock MR, Green MH, Kris-Etherton PM (2011) Effects of adiposity on plasma lipid response to reductions in dietary saturated fatty acids and cholesterol. Adv Nutr 2: 261-274.

28. Vazquez G, Duval S, Jacobs DR, Silventoinen K (2007) Comparison of body mass index, waist circumference, and waist/hip ratio in predicting incident diabetes: A metaanalysis. Epidemiol Rev 29: 115-128.

29. Prior SL, Bain SC, Stephens JW, Alam I, Baxter JN (2009) Total antioxidant status before and after bariatric surgery for type 2 diabetes mellitus. Metabolism 58: 1366.

30. Bala M, Meenakshi, Aggarwal S (2019) Correlation of body mass index and waist/hip ratio with glycated hemoglobin in prediabetes. EJIFCC 30: 317-324.

31. King GL, Loeken MR (2004) Hyperglycemia-induced oxidative stress in diabetic complications. Histochem Cell Biol 122: 333-338.

32. Volpe CMO, Villar-Delfino PH, Dos Anjos PMF, NogueiraMachado JA (2018) Cellular death, reactive oxygen species (ROS) and diabetic complications review-Article. Cell Death Dis 9: 119.

33. Younis NN, Soran H, Charlton-Menys V, Sharma R, Hama S, et al. (2013) High-density lipoprotein impedes glycation of low-density lipoprotein. Diab Vasc Dis Res 10: 152-160.

34. Soran H, Schofield JD, Durrington PN (2015) Antioxidant properties of HDL. Front Pharmacol 6: 222. 